\title{
Une analyse théorisée de données "authentiques»: enseignement du lexique/vocabulaire en littérature au cycle 3
}

\author{
Catherine Dupuy ${ }^{1}$, Stéphanie Genre ${ }^{2}$ \\ ${ }^{1}$ Laboratoire LIRDEF, UM/UPVM, 2 place Marcel Godechot 34092 Montpellier \\ ${ }^{2}$ Laboratoire EMA, UCP, ZAC des Barbanniers avenue Marcel Paul 92230 Gennevilliers, France.
}

Mots clés. lexique/vocabulaire, littérature, construction du sens, gestes professionnels lexique

\begin{abstract}
Résumé. La présente communication cherche à éclairer les activités conjointes portant sur le lexique/vocabulaire en littérature à la fin de l'école élémentaire et au début collège. Pour ce faire, elle s'appuie sur des champs de recherche divers (lexicologie, analyse du discours, didactique de la littérature). Nous centrons notre analyse sur les gestes didactiques de l'enseignant afin d'identifier les interactions les plus déterminantes dans la construction du sens (Rastier, 1987/2009) déroulée dans les mouvements discursifs conjoints (Jeanneret, 1988) des échanges. L'étude menée se base sur plusieurs ensembles de données tous issus du cycle 3 : deux corpus recueillis pour les besoins de la recherche doctorale et des séances de lecture littéraire à l'école recueillies dans le cadre d'une recherche collaborative à l'école. Les «moments lexicaux» ont été choisis pour leur type d'interactions variées (explication, définition, commentaires, proposition de sens ou clarification de l'objet lexical des discussions) et pour leurs ancrages conversationnels (modalisation dans l'interaction, redéfinition de ce dont on parle, compréhension commune, et conduites d'autorégulation).
\end{abstract}

\begin{abstract}
This paper seeks to inform joint activities on lexicon/vocabulary in literature at the end of elementary school and at the beginning of college. To do this, it relies on various fields of research (lexicology, analysis of discourse, teaching of literature). We focus our analysis on the teaching gestures of the teacher in order to identify the most decisive interactions in the construction of the meaning (Rastier, 1987/2009) carried out in joint discursive movements (Jeanneret, 1988) of exchanges. The study is based on several data sets all from Cycle 3: two corpus collected for the purposes of doctoral research and school reading sessions collected as part of collaborative research at the school. The «lexical moments» were chosen for their type of varied interactions (explanation, definition, comments proposed meaning or clarification of the lexical object of the discussions) and for their conversational anchors (modalization in interaction, redefinition of what we are talking about, common understanding, and self-regulation conduct).
\end{abstract}

\section{Introduction}

S'intéressant à d'autres modalités que celles de la définition des mots ou de la recherche du mot juste, cette contribution interroge la diversité des conduites liées au travail sur le lexique/vocabulaire en classe de français/ littérature en isolant les moments où les enseignants du cycle 3 mobilisent l'activité lexique pour faire comprendre et interpréter le texte aux élèves, mobilisant ainsi des gestes didactiques spécifiques que nous appelons « gestes lexique».

On se propose de confronter les pratiques réelles à une mise en perspective didactique qui interroge les procédures favorables aux interventions des enseignants en phase avec les apprentissages des élèves. À partir d'approches disciplinaires complémentaires (lexicologie, sémantique interprétative, didactique du français et de la littérature), l'article mettra en regard les réflexions récentes en didactique du lexique, sur la complémentarité entre enseignement incident et enseignement systématique (Grossmann, 2011), et les pratiques effectives observées.

\footnotetext{
${ }^{1}$ Corresponding author : catherine.dupuy @umontpellier.fr

${ }^{2}$ Corresponding author : stephanie.genre@cyu.fr
} 
Pour cela, nous articulons analyse linguistique de l'unité lexicale (désormais UL) au cœur du maillage vocabulaire/apprentissage du lexique et analyse interactionnelle de l'UL en discours selon des dispositifs empiriques : traitement du sens des mots au fil des échanges, correction, reformulation orale.

Nous commencerons par énoncer les choix théoriques et méthodologiques nécessaires pour étudier les moments lexicaux. Nous expliquerons ensuite la manière dont le corpus d'analyse a été constitué. Nous analyserons enfin les procédures utilisées par les élèves et l'enseignant pour dénouer les nouds didactiques posés par certaines UL du texte à lire.

\section{Nos choix théoriques, méthodologiques et terminologiques}

La question de recherche peut être exprimée à l'aide de la triple interrogation didactique suivante : comment l'objet enseigné lexique se concrétise-t-il dans le cadre d'activités de lecture littéraire ? Dans quelle mesure cet objet croise-t-il des problématiques d'enseignement de la langue et de compréhension/interprétation $\mathrm{du}$ texte? Comment la pratique professionnelle de l'enseignant sélectionnant les UL dans les dits «moments lexicaux » engage à la fois l'élucidation en langue et la subjectivité des sujets lecteurs (Rouxel, 2004). Le choix du corpus de séances de littérature en cycle 3 ouvre donc une perspective sur ce que les élèves construisent avec l'aide de leurs enseignants en termes de développement langagier et de savoirs lexicaux tout autant que de rencontre sensible avec les mots du texte relevant du percevoir/apprécier/analyser (Chabanne, 2002). Ce qui nous intéresse ici c'est d'identifier dans la continuité des travaux de Nonnon (2012), cette « enseignabilité » qui se heurte à la richesse et la difficulté d'un lexique fonctionnant en réseau (Wilks et Meara, 2002 ; Polguère, 2016), les UL d'un texte étant souvent reliées dans l'interaction à d'autres unités selon des relations de différente nature (copolysémie, synonymie, antonymie) ou intégrées dans des structures syntaxiques/sémantiques (collocations).

À travers cet article, nous nous proposons donc d'examiner les multiples facettes de la compétence lexicale (Tremblay, Anctil, Perron, 2016) qui sont autant de noeuds didactiques pour l'enseignant aux prises avec les genres scolaires disciplinaires de la littérature qui font généralement coexister deux grands ensembles de conduites: des demandes d'éclaircissement en situation de communication de lecture (rencontre du vocabulaire du texte avec celui des lecteurs, explication du sens en contexte) et des activités lexique (appui sur la morphologie des mots pour en comprendre la signification : définition des mots en langue hors contexte, utilisation d'une métalangue pour décrire le fonctionnement du lexique, etc.).

Nous utilisons cette dénomination de nœuds didactiques pour signifier que la rencontre lexique/ vocabulaire et littérature est complexe. Nous l'avons choisie d'une part parce que les discussions à thème lexical en littérature concentrent plusieurs problématiques lexicales imbriquées et, d'autre part, car ces dernières posent des problèmes de gestion et d'intervention aux enseignants dans le contexte de la lecture littéraire.

Cette catégorisation sur le plan didactique se décline au niveau interactionnel des pratiques effectives en ce que nous avons nommé les moments lexicaux (Dupuy et Genre, 2020). Dans ces moments lexicaux se retrouvent les conduites attendues de demandes d'éclaircissement et d'activités lexique citées plus haut, mais aussi des conduites qui interrogent la dimension symbolique du vocabulaire. Il serait donc plus juste de parler de moments lexicaux et de vocabulaire pour intégrer cette rencontre du vocabulaire de l'œuvre avec le vocabulaire des lecteurs qu'est la lecture littéraire, mais nous avons choisi de garder cette appellation car notre objet peut se définir comme la mise en fonctionnement de faits lexicaux dans la coconstruction du sens en discours, incluant ainsi travail sur le lexique et travail sur le vocabulaire.

Nous étudions donc à la fois les cheminements discursifs des interlocuteurs en situation de lecture littéraire sur le plan de la sémiotisation, qui fait passer du lexème au vocable, sur le plan de l'élaboration du sens et de la signification (Rastier, 2000, op.cit.) par des communautés discursives (Jaubert, Rebière, Bernié, 2003) dont Plane (2005) a montré qu'elles étaient variées, ainsi que sur le plan de la capacité des élèves, étayée par l'enseignant, à faire jouer la dimension symbolique des mots dans leurs discours.

Le cadre méthodologique quant à lui inclut deux hypothèses. La première avance que, malgré les tâtonnements dans les productions orales cherchant à approcher le sens de l'UL en contexte, il est possible de reconstituer la variété des schémas d'interprétation individuels et collectifs. La deuxième hypothèse cherche à analyser «le circuit» de l'UL au travers des reformulations se présentant sous plusieurs formes: soit l'UL est reprise avec des sens et références variés, soit elle est paraphrasée ou remplacée par des termes équivalents pour les locuteurs.

L'analyse des gestes lexique appuyée sur les verbalisations spécifiques, anticipations, constats et rétroactions autour d'UL permet ainsi de reconstituer la régulation de l'étayage de l'enseignant.

\subsection{Les moments lexicaux : des nœuds didactiques}

Concevoir l'analyse des moments lexicaux dans les interactions verbales sous-tend que l'activité lexique en littérature est une promesse d'apprentissage langagier pour les élèves. Mais la question de 
l'enseignement du lexique (et généralement le domaine de l'étude de la langue et ses autres sous domaines grammaire et orthographe) soulève des zones d'incertitude didactique que les programmes successifs de l'école n'ont pas dissipées. Garcia Debanc, Masseron et Ronveaux (2017) ont à ce sujet montré dans leurs travaux l'intérêt de construire des observations plus fines. C'est ce que nous avons cherché à faire en observant ces nœuds didactiques lexicaux dont nous montrons le caractère polymorphe. Ces derniers pouvant être le lieu de l'explication en langue, de la sensibilité lexicale (Tremblay, 2017, 2018) ou pouvant intégrer encore des éléments de stylistique de vocabulaire. Et c'est cette variété de conduites qui en fait leur caractère problématique et redouté pour l'enseignant qui appréhende tout ce peut s'éloigner d'un travail sur la signification en langue. Il est vrai que la relation " esthétique » aux mots (réceptivité des élèves envers les particularités sonores et graphiques des mots par exemple) s'oppose aux autres formes de travail lexical privilégiées par les programmes scolaires: formation des mots (dérivation, composition), relations (morphologie, synonymie et termes génériques et spécifiques) ou regroupements des mots de la langue (type dictionnaire).

C'est aussi dans le fil des échanges collectifs autour du texte qu'adviennent des « incidents critiques » (Flanagan, 1954). Bien que la compétence professionnelle de l'enseignant repose sur la possibilité de disposer de variantes de routines de métier, des imprévus didactiques (Bucheton, 2008) surgissent inévitablement. Des gestes d'ajustements (Vergnaud, 2001) convoqués par l'enseignant recentrent ses interventions sur ce qui se passe dans les interactions orales car ce sont les tâtonnements autour d'un mot qui génèrent les nœuds didactiques. Parmi les incidents les plus critiques, mais aussi les plus didactiquement prometteurs pour le geste didactique lexique, se trouvent les moments où l'enseignant s'ajuste aux compétences lexicales (Perron, Anctil, Tremblay, 2016, op.cit.) de ses élèves. Ils consistent à orienter le matériau lexical réorganisé successivement au long des échanges en le modifiant sémantiquement et syntaxiquement ou en intervenant à bon escient pour réorienter ou compléter le propos autour des mots, à corriger une interprétation lexicale erronée ou au contraire engager les élèves dans une interprétation lexicale. L'ensemble de ces gestes obligeant à une grande finesse dans l'étayage langagier.

Ajoutons que c'est la variété des stratégies lexicales des élèves plus ou moins hésitantes qui rend l'ajustement complexe et non pas la seule gestion des modèles discursifs utilisés (définitionnel, explicatif, de l'ordre du commentaire ou de la reprise imitative).

\subsection{Les gestes didactiques lexique : une notion convoquée et à construire}

Tout comme la discipline ou les progressions, les gestes professionnels, considérés comme des éléments du système conceptuel de la recherche en didactique du français, au-delà des dissensus et consensus autour de leur polysémie, rappellent que l'activité de l'enseignant, au travers des pratiques réelles dans la classe, est (re)devenue à l'ordre du jour. Alors même que l'on a affaire à un construit théorique bâti autour de problématiques les plus communes comme celle de l'enseignement-apprentissage, partagées par un ensemble de domaines de recherches complémentaires (ergonomie, analyse de pratiques, action conjointe et didactiques comparées), il apparait que la part de la professionnalité conçue comme des pratiques efficaces en situation ne suffise pas à stabiliser les gestes professionnels comme un constituant opérationnel unique des recherches descriptives en français. Quoiqu'il en soit, les spécificités des objets de savoir et des systèmes de représentation propres à la discipline (ici à l'intersection de la langue et de la littérature et par ailleurs les caractéristiques propres du champ conceptuel étudié dans la séquence), nécessite des outils pour produire des analyses reliant les enjeux linguistiques aux enjeux littéraires. (Volteau, Garcia-Debanc, 2008).

Le modèle de l'agir enseignant référence et définit un répertoire de routines et de gestes de métier ajustés par nécessité en contexte. Dans la classe de littérature, la visée d'enseignement organise des formes de travail spécifiques à la discipline. Si le pilotage de la classe (gestion des temps et espaces des dispositifs) et l'atmosphère (les relations entre les élèves, l'ethos de l'enseignant et l'engagement des élèves dans les activités) font partie de la variation de l'action enseignante, nous étudions plus particulièrement l'étayage (Bruner, 1983). Cherchant à mieux décrire l'ajustement des interactions lexique (Kerbrat-Orecchioni, 1990/1998; 2001/2008), afin de dégager un niveau d'étayage d'une nature potentiellement différenciée et variable, nous privilégions les explications lexicales en les dégageant du genre scolaire de l'explication de texte. Nous cherchons à mettre en avant l'élément suivant: faire expérimenter la référentialité des mots dans la langue tout en étayant le décodage des UL par des indices lexico-sémantiques ajustés au processus de construction du sens du texte (Cavalla, 2018) relèverait d'une pratique littéraire spécifique du lexique. Le geste d'étayage lexique selon notre dénomination soulignerait combien la préoccupation didactique du lexique en lecture littéraire relève de l'aide à la compréhension des discours en se polarisant sur l'habileté de l'enseignant à faire apprendre à apprendre des conduites de «l'observation réfléchie et sensible de la langue ». 


\section{Constitution du corpus d'études}

Pour aborder la question de l'enseignement du lexique dans la discipline littérature, nous avons analysé dix séances de lecture littéraire enregistrées en vidéos entre 2010 et 2017 issues de classes de CM2 et de $6{ }^{\text {ème }}$ pour un quart d'écoles et de collèges en zone sensible.

Les premières données ont été recueillies pour les besoins de deux recherches doctorales recensant un certain nombre de conduites spécifiques du lexique/vocabulaire au cours de la lecture littéraire au cycle 3. Le deuxième ensemble de données s'inscrit dans le cadre de la recherche collaborative dont les deux objectifs ont été de rendre compte de l'impact des programmes entrés en vigueur en 2016 sur les pratiques ordinaires des enseignants faisant lire les genres littéraires de l'école (romans poésie théâtre et bandes dessinées). Cette recherche ayant pour cadre méthodologique un cadre multi-référencé d'analyse : analyse didactique et langagière des interactions entre élèves, analyse des gestes professionnels de l'enseignant et de recueillir un ensemble de données qui rassemble plus d'une vingtaine de séances filmées en CM2 et $6^{\text {ème }}$, des entretiens d'autoconfrontations (Theureau, 2006), et des entretiens croisés avec et entre les enseignants.

Les extraits de séances issus des deux corpus distincts dont nous venons de parler sont suffisamment significatifs pour mener une description compréhensive des choix didactiques lexique effectués par les enseignants. Pour le corpus 1 l'objet d'étude focalise sur les processus collectifs et individuels de construction du sens au travers des nœuds didactiques lors d'épisodes explicatifs qui relèvent d'obstacles divers (lexicaux, linguistiques, thématiques et inférentiels). Nous analysons d'abord le travail réel de maîtres expérimentés faisant expliquer chacun à leur manière des UL identiques d'une nouvelle de littérature de Jeunesse : Archimémé, extraite d'un recueil de Littérature Jeunesse de Bernard Friot paru en 1999 aux Editions Milan.

Un autre exemple ensuite dans le corpus 1 décrira un épisode sur l'UL marâtre dans une séance de lecture de l'album La Petite fille du livre de Nadja (l'École des loisirs, 1999) s'intéressant aux caractéristiques propres des modalités de l'ajustement. À la suite de ces analyses, nous aurons l'occasion de recenser et de comparer les gestes lexicaux mobilisés dans les deux analyses.

Le corpus 2 servira quant à lui d'appui pour observer les échanges autour de nœuds didactiques lorsque les énoncés de réception des élèves font intervenir la dimension symbolique lexicale dans leurs discours. Nous parlerons ainsi des mots de la réception arbre de roses à propos de La belle et la bête et mère à propos de La Petite fille du livre qui sont tous deux des indices de l'interprétation sensible des élèves.

La sélection des moments lexicaux s'est faite sur la base d'épisodes que nous définissons comme des unités d'activité métalexicale circonscrites dans des périodes mono et/ou polylogale correspondant dans le cours des échanges de la lecture littéraire à des discussions autour des mots (du texte ou de la réception). Leur découpage n'a pas été toujours évident d'autant qu'ils peuvent être « éclatés » dans la séance (Genre, 2018). Nous avons pu rencontrer des difficultés à extraire sur un plan didactique les tâtonnements (reprises et reformulations des interlocuteurs) dans la construction du sens à propos de termes du texte à lire. C'est pour cela, que nous choisissons d'exposer les épisodes les moins problématiques en termes de repérage et d'identification de l'activité méta lexicale, sans viser à l'exhaustivité, mais en voulant décrire et comprendre les orientations et enjeux généraux des moments lexicaux en littérature à partir des unités d'analyse de notre modèle.

\section{Analyse du corpus}

\subsection{Quelles formes interactionnelles prennent les explications lexicales sur la caractérisation d'un personnage?}

Les deux séances retenues du corpus 1 (classe A et classe B) ont porté sur la même nouvelle lue à des élèves du cycle 3 dans les classes de zone sensible. C'est le récit d'une arrière-grand-mère Archimémé qui bien qu'effrayante attire la complicité de son petit-fils Baptiste. Les enseignants ont privilégié pour la lecture compréhension de se focaliser sur le portrait du personnage de l'aïeule bâti sur une série d'UL qualificatives renforçant l'extrême vieillesse de celle-ci qui surdétermine le rôle du personnage de jeune garçon inquiet par rapport à son arrière-grand-mère. Nous examinons avec des extraits de deux séances trois UL, supports de discours explicatifs produits et partagés dans les échanges : voix fade et usée, voix geignarde et rire grelottant. Sur cette base on décrit comment se déroule à l'oral le processus d'essais et d'hésitations inhérent au processus de l'explication (Hudelot, 2001).

3.1.1 Guidage discursif de l'explication lexicale dans la classe A : les UL voix fade et usée et voix grelottante 
Dans le premier épisode choisi, les élèves de la classe A sont amenés à travailler en groupe à la lecture expressive des extraits du texte. L'enseignant incite ses élèves à faire des propositions d'explicitation à propos de voix fade et usée et voix grelottante.

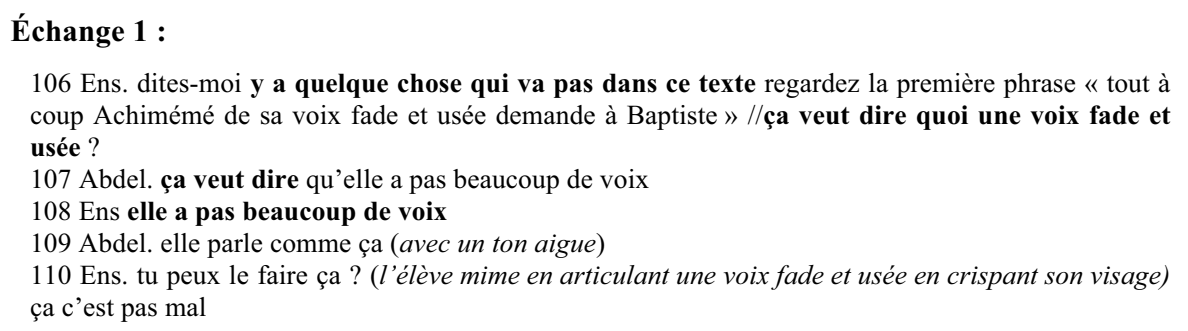

Focalisant leur attention sur le sens en contexte, l'enseignant signale ce qu'il présente comme une anomalie : "y a quelque chose qui va pas dans ce texte». Son geste lexique consiste ici à attirer l'attention des élèves sur les UL fade et usée en relisant une partie du texte, ce qui permet de réinsérer les UL dans leur cotexte. La reprise explicative d'Abdel : "ça veut dire qu'elle a pas beaucoup de voix » démarre une séquence « multimodale » portant sur la reformulation verbale de l'UL à laquelle s'ajoute un engagement prosodique 109 et gestuel 110 que l'enseignant valide.

Dans un autre extrait, à propos de l'UL voix grelottante cette fois, le guidage discursif de l'enseignant repose en premier lieu sur le même format d'interaction appelant l'explication de l'UL : « qu'est-ce que ça veut dire une voix grelottante ? ». Toutefois celui-ci ajoute un geste lexique qui consiste à inciter ses élèves à actualiser le sème afférent /vieillesse/.

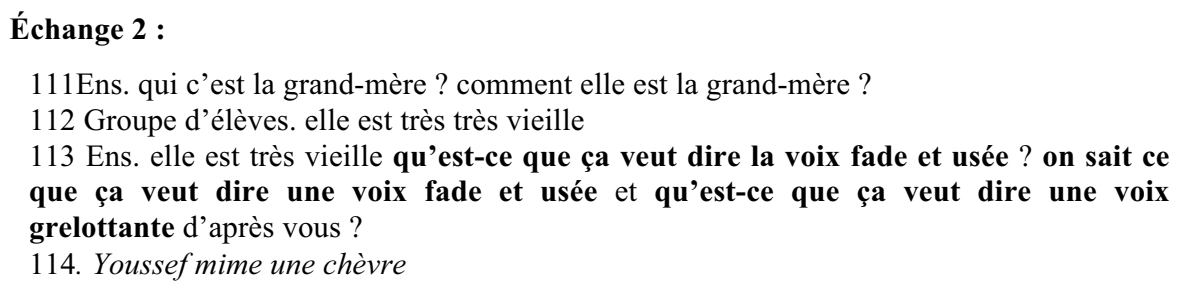

Si dans cette classe A, le guidage discursif a porté sur voix fade et usée et voix grelottante, le choix de l'enseignant de la classe $\mathrm{B}$ n'est pas tout à fait identique. Cette mise en regard de pratiques est intéressante dans la mesure où l'on voit bien que la thématisation de telle ou telle UL engage des chemins explicatifs et/ou interprétatifs différents et c'est ce que nous allons voir à présent.

\subsubsection{Guidage discursif de l'explication lexicale dans la classe B: les UL rire grelottant et voix geignarde}

Dans le premier des épisodes lexicaux de la classe B, les échanges portent d'abord sur rire grelottant (et non pas voix grelottante utilisée par l'enseignant de la classe A).

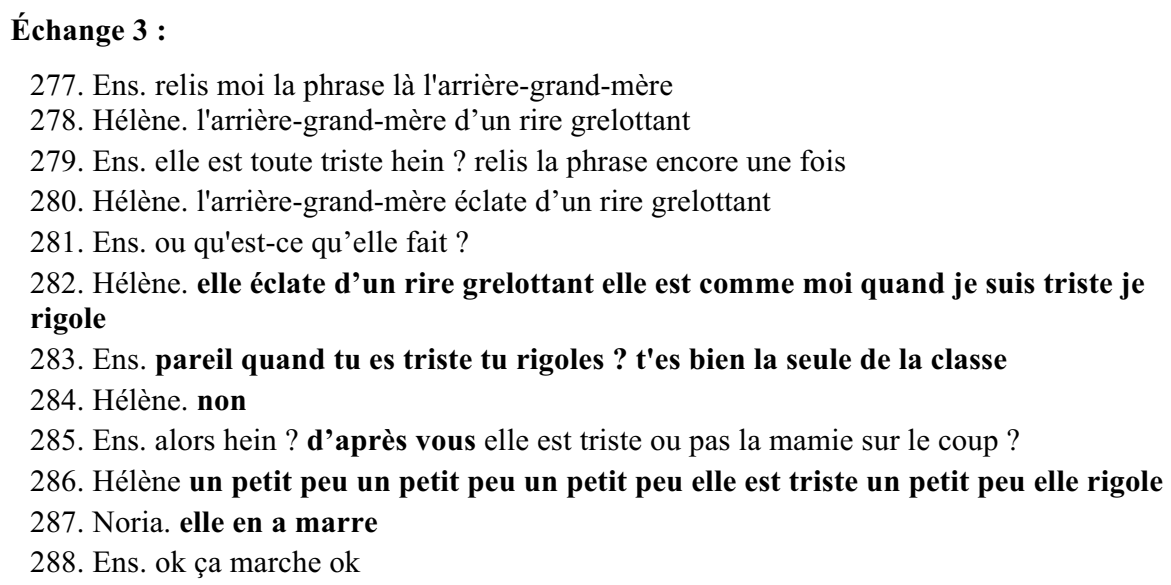

À la demande de l'enseignant, Hélène relit l'extrait plusieurs fois à partir de 278. Cette reprise in extenso de l'énoncé du texte dans les trois tours de parole successifs par Hélène relève de la difficulté liée à la compréhension de l'expression métaphorique rire grelottant qui ne pose pas réellement de problème 
sémantique mais plutôt une difficulté syntagmatique due à l'expression. C'est bien là l'association des deux termes qui est problématique.

$\mathrm{Si}$ on pouvait s'attendre à ce que l'enseignant cherche à faire reformuler l' ensemble des élèves pour expliciter les sens de la métaphore (De Gaulmyn, 1991), il n'en fait rien et préfère rebondir sur l'intervention d'Hélène en 282 qui donne une équivalence de sens tirée de sa propre expérience. La prise de parole qui clôt l'épisode : « un petit peu un petit peu un petit peu elle est triste un petit peu elle rigole » montre une tentative d'essai explicatif de la part d'Hélène qui ne s'appuie pas sur la recherche de synonyme mais présente une auto reformulation basée sur une modalisation atténuative. Il semble que le propos de cette élève ait engendré la compréhension de rire grelottant. Celle-ci fait référence aux sèmes opposés /tristesse/et /joie/ qui, à un niveau abstrait (métaphorique), pourrait correspondre au sens de tremblement ou de bouleversement émotionnel. Mais l'enseignant repousse l'explication fondée sur des savoirs d'expérience : « quand tu es triste tu rigoles ? t'es bien la seule de la classe » ne choisissant pas de s'appuyer sur une intervention hétéro-reformulée qui aurait pu être partagée avec les autres élèves. L'absence ici de geste d'atmosphère (Bucheton, Soulé, 2009) de la part de l'enseignant ne semble pas être un frein à la discussion puisqu'Hélène s'engage tout de même dans la recherche du sens.

Plus loin dans la séance de cette même classe B, l'enseignant ouvre un autre épisode d'explication lexicale ayant comme thème une voix geignarde.

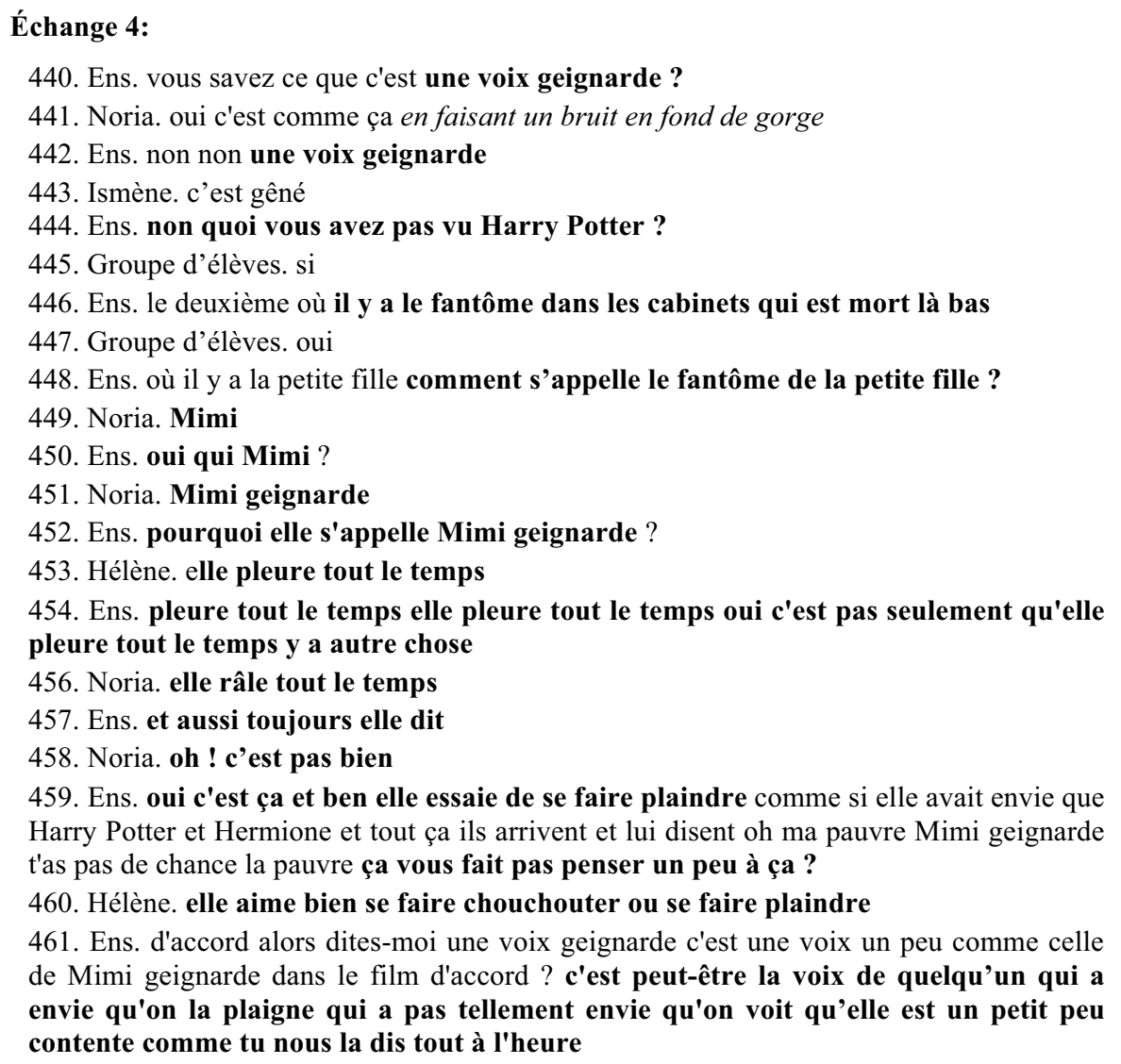

La dynamique des interactions amorcée par un rejet des propositions de deux élèves repose sur la question inaugurale de l'enseignant à propos d'Harry Potter. Son intonation différente exagérément étonnée ouvre l'épisode basé sur un cheminement hypothético-déductif auquel se prête volontiers la classe. L'enseignant, par l'intermédiaire de questions sur les souvenirs du film que la classe dit avoir vu, délivre les indices nécessaires pour qu'un élève nomme le personnage concerné : Mimi la Geignarde. Il engage les élèves à réfléchir sur l'attribution de ce patronyme. Sollicitant les propositions d'explication, l'enseignant semble suivre les mouvements discursifs conjoints de deux élèves (Jeanneret, 1988) qu'il accompagne par une relance et une confirmation positive, convoquant cette fois un geste d'atmosphère (Bucheton, Soulé, op.cit.) propice à l'engagement des élèves dans la recherche du sens. Il reprend une partie de l'énoncé 460 : « Oui c'est ça et ben elle essaie de se faire plaindre ». En donnant l'impression de se laisser influencer par les interactions, l'enseignant semble prendre conscience du problème posé par cette UL peu usuelle. En fin d'extrait, celui-ci entre dans un discours explicatif en hétéro-formulation qui inclut les propositions des deux élèves et, en citation, l'énoncé antérieur d'Hélène : « elle est un petit peu 
contente comme tu nous la dis tout à l'heure ». L'épisode s'est déroulé selon des interactions fluides sur le mode conversationnel alors que le sens de l'UL à chercher contenait des traits sémantiques abstraits. Lors de l'autoconfrontation, l'enseignant semble avoir été conscient de ce moment lexical même s'il maintient que : «quand tu expliques tout de façon après tu repasses de toute façon tu as des réajustements à faire je vais être obligé parce que eux ils partent ailleurs ».

Voici ce que nous pouvons dire à présent sur la gestion des épisodes précédents par les deux enseignants : ces derniers ont cherché à élucider le sens d'adjectifs peu usuels fade et usée / grelottant et geignarde à partir de la structure Nom + adjectif. Le fait que ces derniers soient associés aux termes courants voix et rire a activé des interactions explicatives de la part des élèves. Elles se sont réalisées sous des formes verbales et gestuelles, ce qui marque la sensibilisation des élèves aux potentialités des procédures de compréhension qui peuvent les faire cheminer vers celles de l'interprétation.

C'est ce type d'interactions entre le texte fictionnel et le lecteur, incluant même la dimension corporelle de la réception, qui fait intervenir selon nous l'expérience lexicale littéraire comme approche sensible.

\subsubsection{Des ajustements à base de reformulation comme voies d'explication pour caractériser Archimémé}

On observe par ailleurs des gestes d'ajustement lors d'épisodes d'explications lexicales. Afin de lever des nœuds didactiques, chacun des deux enseignants des classes A et B s'appuie sur les usages de la coréférence dans les manifestions linguistiques et discursives (Mortureux, (1997/2013) et sur la reformulation (Gülich et Kotschi, 1987).

Alors que la voix fade et usée a été expliquée, l'enseignant de la classe A reprend la désignation de grand-mère pour enchainer sur l'élucidation du sens de voix grelottante comme ci-après : « ça veut dire qu'elle a pas beaucoup de voix elle a pas beaucoup de voix (rephrasage 1 de l'enseignant).

Dans ces deux énoncés, la coréférence est structurée dans le même calque sémantique et syntaxique que celui des énoncés des élèves: " elle est très très vieille elle est très vieille » (rephrasage 2 de l'enseignant). À cela s'ajoute un autre ajustement de l'enseignant qui consiste à établir le parallèle entre fade, usée et grelottante de manière à faire ressortir le sème /vieillesse/.

$\mathrm{Au}$ cours de l'épisode sur voix geignarde, l'enseignant de la classe B mise sur les connaissances extralinguistiques des élèves pour ensuite ajuster les formes interactionnelles de l'explication. Cela permet aux élèves d'associer les sèmes afférents /pleur /et/plainte/ à geignarde mais sans valeur dépréciative. Le nœud didactique pris en charge par l'enseignant à propos de cette association de sens se joue dans la séquence d'hétéro-reformulation (la reformulation du propos des apprenants) qui suit. Il déploie au fur et à mesure la co-construction du sens en la négociant dans une séquence structurée par la coréférence. Il apparait que l'organisation des enchainements d'énoncés développe une reformulation en deux temps. À partir du sème afférent /pleur/ évoqué par l'élève, l'enseignant enchaine par un premier rephrasage. M. « elle pleure tout le temps elle pleure tout le temps oui c'est pas seulement qu'elle pleure tout le temps y a autre chose ». Un second intervient ensuite : « oui c'est ça et ben elle essaie de se faire plaindre " auquel succède celui d'un élève : " elle aime bien se faire chouchouter ou se faire plaindre ». Ces rephrasages (Gülich et Kotschi, 1987, op.cit.) successifs semblent avoir pour chacun des interlocuteurs une fonction commune : celle de maintenir la dynamique de communication.

En ce qui concerne le dernier énoncé de l'enseignant de la classe B « une voix geignarde c'est une voix un peu comme celle de Mimi geignarde dans le film d'accord ? c'est peut-être la voix de quelqu'un qui a envie qu'on la plaigne », celui-ci met en évidence le principe de la reformulation entre segment à reformuler voix geignarde et segment reformulant en discours « la voix de quelqu'un qui a envie qu'on la plaigne $»$.

Les échanges des deux enseignants et de leurs élèves ont consisté ainsi à investir, surtout pour l'enseignant $\mathrm{B}$, la charge culturelle (Genre, 2015) du stéréotype de l'arrière-grand-mère. Tous deux ont traité le noyau sémantique des UL ajusté aux essais ou tâtonnements discursifs du cheminement de sens porté par les reformulations en contexte.

\subsubsection{Des ajustements à base de propositions différentielles pour l'explication de marâtre}

Dans le cas présenté (classe $\mathrm{C}$ ), la séquence constituée d'échanges a pour acte directeur (KerbratOrecchioni, (2001/2008) l'UL marâtre :

77. Ens. elle se fait battre qui la bat - dans Cendrillon

78. E. sa belle-maman

79. Ens. sa quoi sa belle-mère ou sa mère

$[\ldots]$

83. Ens. qu'est-ce qu'est une marâtre

84. E. c'est la belle-mère c'est la c'est la 


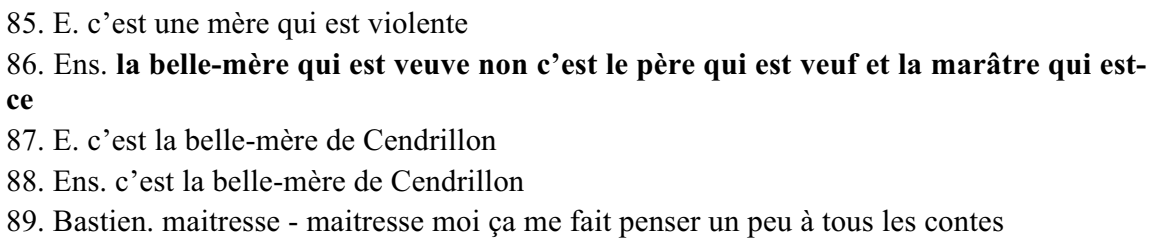

L'épisode, comme nous avons pu le dire en amont, pose des problèmes de délimitation. La question de son bornage dépend de deux facteurs: l'un davantage sémantique, l'autre davantage linguistique. En effet, si l'on tient compte de l'apparition du contenu sémantique du vocable, on peut tout à fait considérer que l'épisode débute aux tours de parole 78 et 79 . En revanche, si l'on privilégie la dimension linguistique en se basant sur la présence du marqueur interrogatif «qu'est-ce que » accompagné d'une question de clarification « méta-lexicale » (Lüdi, $1991: 206$ ), on préfèrera une ouverture de l'épisode au tour de parole 83. Quoiqu'il en soit, ce qui nous intéresse dans cet épisode, c'est bien le geste lexique contrastif de l'enseignant au tour de parole 79 («sa quoi sa belle-mère ou sa mère») associé à réorientation d'ordre sémantique («non c'est le père qui est veuf») qui pousse d'abord ses élèves à opérer une définition différentielle pour ensuite préciser quelle l'UL associée au veuvage. L'échange se déroule ainsi :

\section{A c'est B donc ce n'est pas C + c'est D + exemplification}

Autrement dit : \{la marâtre $\}$ "c'est » \{la belle mère $\}$ donc «ce n'est pas » $\{$ 《la mère »\} + «c'est » \{ « une mère qui est violente " + «c'est la belle-mère de Cendrillon »\} avec un élargissement au genre «conte»: \{ «moi ça me fait penser un peu à tous les contes »\}. Si l'on en croit F. Grossmann, l'apprentissage du vocabulaire est bien lié à la question du choix (Grossmann, 2003) et c'est ce dont témoigne l'extrait présenté. Une des manifestations du geste lexique pourrait être ainsi nommée : «geste lexique d'aide au choix ».

Les conduites analysées jusqu'à présent concernaient des moments lexicaux d'explication pourrait-on dire «classiques », (reliées au genre professionnel de l'explication des mots) celles à venir cernent d'autres enjeux qui relient le vocabulaire à l'interprétation de l'œuvre.

\subsection{Nœuds didactiques dans la prise en compte des mots de la réception des élèves}

Nous poursuivons notre questionnement sur les contenus et les formes des interactions langagières susceptibles de se constituer en nœuds didactiques. Parmi ces derniers, d'autres épisodes recouvrent ce que nous appelons les mots de la réception. Dans ces épisodes, les traces langagières de la langue et du discours correspondent à des pratiques interprétatives du vocabulaire par les élèves. Ce que nous avons défini comme le sens pour soi (Genre, 2015) rassemble ainsi des phénomènes de subjectivation (engagement subjectif, expérience esthétique, projection du lecteur, activation fantasmatique, projection/introjection).

Le corpus 2 (classes D et E) sert donc d'appui pour observer les énoncés de réception des élèves qui font intervenir la dimension symbolique dans leurs discours. L'ajustement de l'enseignant consiste donc ici à réagir aux commentaires des élèves qui expriment des valeurs, des appréciations et/ou des affects.

L'analyse des mots de la réception revient à repérer la manière dont les connaissances lexicales des élèves sont mobilisées dans les mouvements interactionnels. Si l'enseignant a pu préparer la séance en retenant les mots qui guident ses élèves vers une compréhension commune, il est cependant plus malaisé pour lui de s'ajuster en prenant des décisions en temps réel qui puisse accompagner au mieux les commentaires des élèves à propos des mots.

\subsubsection{Les mots de la réception : le vocabulaire comme indice de la symbolique de l'œuvre}

Nous avons choisi, pour illustrer cette dynamique d'entrée dans le sens, une séance de lecture du conte $\mathrm{La}$ belle et la bête (classe D) autour de 1'U.L. berceau de roses car elle est révélatrice d'enjeux: sémantiques, intersémiotiques (réception du texte de Leprince de Beaumont en lien avec les connaissances des élèves sur les dessins animés et films) mais aussi littéraire (sur la culture scolaire du conte). En accord avec ces déclarations en autoconfrontation, «l'école a quelque chose à faire [...] pour élever le débat, la sensibilité le sens profond des choses ", l'enseignant annonce aux élèves que : "là on va vraiment s'occuper des mots du texte » à propos du passage où le père de La Belle découvre dans le château de la Bête un jardin rempli de roses.

$\mathrm{Au}$ début de la séance, l'enseignant intervient pour orienter les échanges sur berceau de roses présent dans l'extrait lu : 
90. Ens. Ben dans la cour c'est dehors aussi à quel endroit est ce ça se passe précisément alors je vous ai résumé brièvement c'est vrai ce qui se passe juste avant l'extrait rappelez-vous il est dans sa chambre enfin il est dans le palais et il voit par la fenêtre il voit quelque chose par la fenêtre que voit-il ?

91. Thomas. euh un arbre de roses

92. Ens. euh je vous relis

La réponse de Thomas « un arbre de roses » n'est pas retenue par l'enseignant. Pourtant, l'équivalence sémantique arbre/berceau semble acceptable dans le contexte. Elle aurait pu servir à amorcer une discussion sur la symbolique du berceau de roses puisque le parasynonyme utilisé par l'élève, fortement lié au contexte, aurait pu être associé au jeu intersémiotique de l'illustration, ouvrant la voie à une modalisation affective et esthétique. L'enseignant aurait pu s'ajuster à ce mot de la réception de manière à faire partager à l'ensemble des interlocuteurs la charge culturelle (Genre, 2015, op.cit) et symbolique du conte.

L'enseignant préfère demander une explication de l'UL à laquelle Raphaël se soumet volontiers :

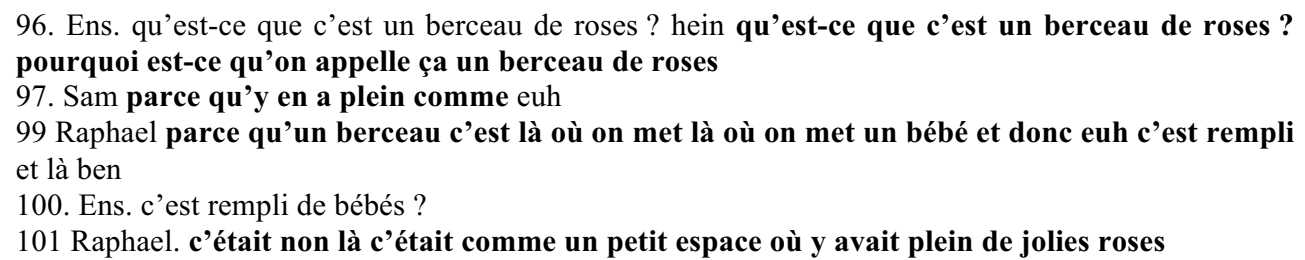

Dans cette classe de Cours Moyen l'UL berceau de roses est alors expliquée par l'enseignant de manière référentielle, alors que le processus énonciatif de Raphaël «c'était comme un petit espace où y avait plein de jolies roses » relie réception du sujet lecteur (Rouxel et Langlade, 2004) et symbolique de l'UL rose. Symbolique sur laquelle l'enseignant revient a postériori dans l'autoconfrontation (Theureau, 2006, op.cit.) : «pour le coup le «berceau» ça peut aussi être symbolique puisque c'est la naissance la naissance de l'histoire la naissance de voilà d'un amour futur etc. mais je vais pas non plus chercher le symbolisme partout c'est aussi pour ça que je laisse tomber et je passe à autre chose ».

\subsubsection{Les mots de la réception : le vocabulaire comme indice de projection dans l'œuvre}

Nous allons présenter pour finir un extrait de La Petite fille du livre (classe E) dans lequel un élève associe le personnage de la femme écrivain à la «mère» de la petite fille, activant ainsi le sème /protection/. Cette interprétation est particulièrement intéressante puisque dans cet album l'auteur, Nadja, offre aux jeunes lecteurs une histoire symbolique de "livre dans le livre » dont la fin est problématique pour des élèves de cycle 3 car les personnages des deux histoires se retrouvent dans une seule et même narration: l'écrivain de l'histoire est-elle la mère de la petite fille ? Créatrice, est-elle la mère de l'œuvre? Le texte, en pratiquant l' «ambiguïté anaphorique » est "délibérément problématique » (Tauveron, 2005: 63) et proliférant (Tauveron, 2005) car il ne décide pas d'une seule et même interprétation :

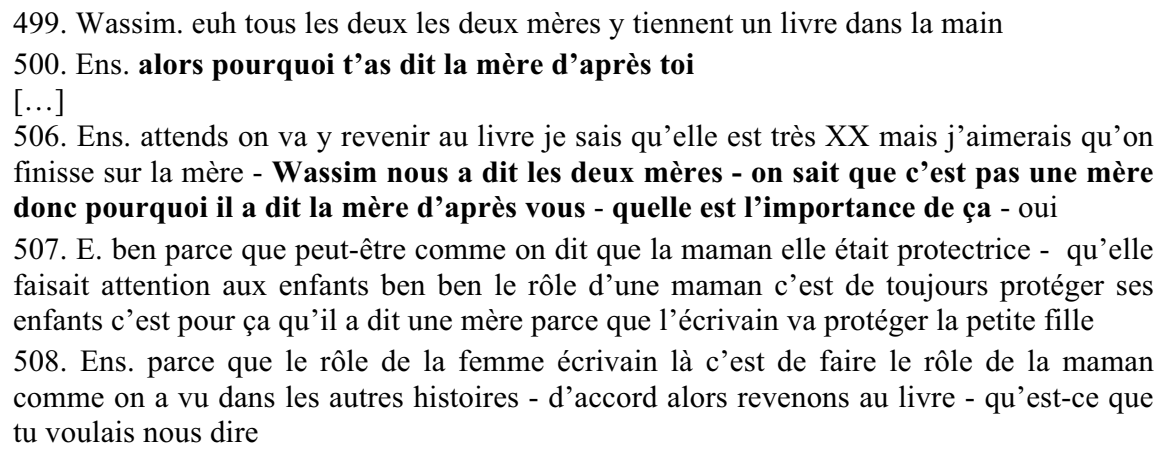

Le geste lexique de l'enseignant procède ici d'un «questionnement lexique ouvert » qui consiste à demander à l'élève d'expliciter un de ses mots de la réception. Celui-ci insiste auprès de son élève pour que ce dernier exprime sa propre " activité fantasmatique » (Langlade, 2008) en lui demandant de dire pourquoi il utilise maman lorsqu'il parle de l'écrivain. Cette attention aux mots de la réception est particulièrement intéressante en littérature car ce sont bien les mots utilisés par les élèves dans leur commentaire qui sont des indicateurs de l'interprétation de l'œuvre. 
À travers cet exemple, nous souhaitons mettre en avant ce geste lexique parce que celui-ci s'éloigne des routines d'élucidation du sens des mots dans le texte et qu'il laisse la main aux élèves s'extrayant ainsi du contrôle de l'enseignant.

\section{Constats et Résultats}

De manière générale, on voit par les exemples tirés des deux corpus de séances de lecture littéraire comment les enseignants sont amenés à exploiter les potentialités des moments lexicaux propres à l'explication lexicale en interaction et sont conduits à réaménager les échanges afin de se réapproprier les énoncés des élèves pour contribuer à la compréhension et interprétation du vocabulaire du texte à lire. L'analyse de ces deux types de conduites nous a permis de restituer les processus d'inscription discursive au service de la compréhension du texte par la formulation du discours explicatif au fil des échanges entre enseignants et élèves.

Nous voulons maintenant établir une synthèse sur les nœuds didactiques comme obstacles présents dans les moments lexicaux. Pour cela, nous soulignons une des propriétés des explications lexicales présentes dans les verbatims constituée selon une double ligne de force contradictoire et complémentaire. La première, inhérente à l'orientation de l'interaction vers une finalité externe (Jacques, 1993), c'est-àdire vers une finalité dépassant le seul souci de la relation pour viser des savoirs, oppose les stratégies discursives de l'enseignant à celles des élèves pris dans les échanges conversationnels.

La deuxième complémentaire au niveau de l'orientation de l'interaction tend vers une finalité interne qui valorise les phénomènes d'explicitation pris en charge en individuel par les stratégies discursives des élèves. Celle-ci pouvant intégrer une dimension argumentative (manifestation de désaccord) étant donné que le dispositif de lecture s'inscrit dans les cadres d'un débat à visée littéraire.

Nous concevons alors que l'engagement dans les explications lexicales puisse correspondre chez les élèves à des pratiques dites argumentatives travaillées discursivement à partir des UL thématisées. Pratiques intermédiaires entre explication et argumentation car on suppose que les élèves n'en maitrisent pas totalement les mécanismes et qui se présentent sous la forme de discours d'exploration actualisés par des associations lexicales spontanées ou réfléchies.

On étudie par deux exemples ci-dessous des stratégies à l'œuvre pour noter la part significative de la gestion de l'étayage enseignant qui consiste à placer les élèves dans des stratégies sémantiques tournées vers l'obtention de l'intercompréhension.

\subsection{Les nœuds didactiques révélateurs des stratégies discursives de l'étayage explicatif} Le point de vue que nous avons tenu a priori tient toujours. " Le discours didactique est en relation privilégiée avec l'explicatif » (Halté, 1988: 7). C'est par conséquent la gestion du discours explicatif recouvrant les compétences discursive et communicationnelle qui est primordiale. On considère alors que l'étayage explicatif repose sur les formes d'organisation du discours de l'aide à la compréhension. Celles -ci servent de support aux intentions de l'interlocuteur qui se déclinent en exemplifications et en reformulations.

On peut ainsi résumer quelques échanges décrits précédemment de manière à visualiser quelques organisations possibles de l'explication lexicale. Ainsi pour le personnage Archimémé la stratégie discursive se présente en trois mouvements. Le premier mouvement ouvert par l'enseignant pose l'explication comme la résolution d'un problème à laquelle s'ajoutent deux explications enchainées.

\section{Signalisation d'un problème dans le texte : initiative de l'enseignant}

106 Ens. dites-moi y a quelque chose qui va pas dans ce texte regardez la première phrase «tout à coup Achimémé de sa voix fade et usée demande à Baptiste »

\section{Démarche d'explicitation (1): demande d'explication de l'enseignant, verbalisation d'un élève \\ 106 bis Ens. ça veut dire quoi une voix fade et usée ? \\ 107 Abdel. ça veut dire qu'elle a pas beaucoup de voix) $108 \mathrm{M}$ elle a pas beaucoup de voix}

3. Démarche d'explicitation (2): demande d'explication de l'enseignant, modalité non verbale d'un élève

109 Abdel. elle parle comme ça (avec un ton aigue)

110 Ens. tu peux le faire ça ? (l'élève mime) ça c'est pas mal

Une autre organisation de l'explication lexicale consiste à sélectionner une partie du texte pour attirer l'attention des élèves et engager une discussion davantage argumentative. 
1. Démarche d'explicitation: demande d'explication de l'enseignant : mise sur la voie avec le balisage sur la phrase ; lecture à haute voix d'un élève

277. Ens relis moi la phrase là l'arrière-grand-mère

278. Hélène. l'arrière-grand-mère, d'un rire grelottant

279. Ens elle est toute triste hein ? Relis la phrase encore une fois

2. Résolution par l'élève : positionnement explicatif et argumentatif : exemplification et comparaison

282. Hélène. elle éclate d'un rire grelottant elle est comme moi quand je suis triste je rigole

3. Rappel à l'explicitation : minimisation de l'explication-argument par l'enseignant

283. Ens. pareil quand tu es triste tu rigoles ? T'es bien la seule de la classe

4. Négociation argumentative «douce» par l'élève : alignement affiliatif envers l'enseignant et auto reformulation paraphrastique

286. Hélène un petit peu un petit peu un petit peu elle est triste un petit peu elle rigole

À partir de ces schématisations on peut voir que les enseignants optent soit pour des conduites explicatives des plus classiques qui, relevant de routines professionnelles sont généralement anticipées par les enseignants (énonciation d'un problème pour questionner, résolution puis conclusion). Soit, pour des conduites davantage argumentatives qui nécessitent un ajustement aux discours justificatifs lexicaux des élèves en situation qui peuvent laisser la part aux imprévus.

\subsection{Les nœuds didactiques comme enchevêtrement de la parole singulière et de la parole collective}

L'ensemble des analyses proposées montre aussi la complexité des moments lexicaux parce que ceux-ci croisent l'explication collective et l'explication singulière, parce qu'ils peuvent être aussi des lieux de dissensions entre le choix de l'enseignant et la tournure conversationnelle autour des UL thématisées.

Ils sont très souvent difficiles à gérer pour l'enseignant dans la mesure où ils sont le lieu à la fois de l'anticipation et de l'imprévu, du contrôle et du lâcher-prise.

Ainsi dans les extraits relatifs à la Petite fille du livre, on voit bien que cette parole singulière de la femme-écrivain considérée par un élève comme la mère du personnage de la petite fille initie l'interprétation collective grâce au geste de lâcher-prise de l'enseignant qui questionne l'élève sur le mot de la réception qu'il utilise.

Dans l'explication de marâtre en revanche, l'enseignant, même s'il rebondit sur marâtre, mot de la réception d'un élève, reste focalisé sur la sémantique alors que ses élèves sont aux prises avec la dimension symbolique du personnage qu'il rattache au genre du conte.

\subsection{Quelques pistes didactiques nourries par les pratiques réelles}

Les moments lexicaux observés dans cet article ont montré tout à la fois leur richesse et leur caractère complexe. Nous avons pu, au fil des analyses, extraire et sélectionner chez les enseignants des gestes professionnels langagiers ainsi que des gestes ancrés dans le genre scolaire de l'explication de texte qui permettent selon nous de dénouer quelques-uns des nœuds didactiques rencontrés dans l'apprentissage incident du lexique/vocabulaire au cours de la lecture littéraire.

Nous nous proposons ici de les traduire sous forme de propositions qui puissent être transférées et devenir généralisables pour toute séance de lecture littéraire, au-delà même de la singularité des œuvres et de la diversité des acteurs. Les différentes interventions que nous avons répertoriées et que nous choisissons de mettre en avant partagent la caractéristique commune de solliciter le locuteur élève et d'apporter une attention aux mots qui n'est pas exclusivement référentielle.

\subsubsection{Les gestes du corpus, des gestes transférables au-delà des séances observées}

Ces gestes pourraient être formulés auprès des professeurs d'école en formation initiale comme en formation continue comme suit avec, signalés entre parenthèses les enseignants du corpus et les notions linguistiques et/ou enjeux associés :

- faire porter l'attention sur certains mots du texte (l'enseignant de la classe A, à propos des UL fade et usée; mots du texte, moment lexical comme une résolution de problèmes qui sert la compréhension/interprétation de l'œuvre).

- faire parler l'entourage des mots expliqués (l'enseignant de la classe A, à propos des UL fade et usée ; mots du texte, notion linguistique de cotexte pour inférer le sens). 


\section{- faire parler l'élève :}

a) pour l'inciter à parler de ses propres mots (l'enseignant de la classe $\mathrm{E}$, à propos de UL mère ; mot de la réception comme indice de projection dans l'œuvre).

b) pour l'inciter à faire des relations entre les mots, (l'enseignant de la classe $\mathrm{A}$, à propos des UL fade, usée en relation avec le sème /vieillesse/ de l'UL grand-mère; mots du texte, lexique en tant que réseau de relations, ici sémantiques);

c) pour l'inciter à faire des relations entre les mots et le monde (l'enseignant de la classe $\mathrm{B}$; à propos de UL geignarde; mot du texte, lien sémantique et connaissances extralinguistiques)

d) pour l'inciter à choisir entre plusieurs propositions (l'enseignant de la classe C; à propos de UL marâtre; mot de la réception, ajustement sémantique dans l'interaction à partir de propositions différentielles)

Ces gestes devront être énoncés explicitement aux élèves sous forme de stratégies lexicales comme le préconisent bon nombre de didacticiens du lexique dont Anctil, Singcaster et Tardif (2018) se font l'écho. Pour ne s'en tenir une fois encore qu'aux gestes ci-dessus, extraits d'un corpus réduit, voici comment nous imaginons leur possible présentation aux élèves :

- Quand je cherche le sens d'un mot ou de plusieurs mots rapprochés dans un texte, je regarde autour de ce mot les mots qui peuvent m'aider ou me donner des idées pour le comprendre ;

- Quand je cherche le sens d'un mot ou de plusieurs mots rapprochés dans un texte, je peux chercher dans ma tête les mots de sens voisin;

- Quand je cherche le sens d'un mot ou de plusieurs mots rapprochés dans un texte, je peux chercher dans ma tête ce à quoi me fait penser le mot ou les mots rapprochés; je peux penser par exemple à des livres, des films, à tout ce que je connais ;

- Quand je cherche le sens d'un mot ou de plusieurs mots rapprochés dans un texte, je peux aussi dire ce que je ressens.

Il ne s'agit-là que d'une indication puisque bien évidemment ces stratégies doivent être énoncées par les élèves dans chaque classe avec leurs enseignants. Quoiqu'il en soit, ce n'est qu'à cette condition qu'il leur sera possible d'avoir une conscience lexicale (Graves 2006, cité par Anctil, Singcaster, Tardif, 2018, op.cit.). Ce rapport à la langue conscient leur permettra de savoir qu'ils peuvent être confrontés à un problème d'ordre lexical mais aussi, et nous insistons sur ce point, qu'en littérature, le rapport aux mots peut être de l'ordre du sensible et du symbolique (voir dans notre corpus notre proposition pour la classe $\mathrm{D}$ et l'UL berceau de roses, et pour la discussion de la classe E autour de l'UL mère). Bien qu'interrogé parfois, celui-ci demeure sous-exploité par les enseignants qui privilégient les réponses directes à des besoins lexicaux ponctuels (Dreyfus, 2004, Cèbe, Goigoux, 2013 ; Anctil, Singcaster, Tardif, 2018, op.cit.) alors même que cette "sensibilité lexicale" (Tremblay, 2017) fait partie pour nous de la compétence lexicale en littérature. Il nous semble important d'attirer l'attention sur le fait qu'une préoccupation louable, celle de ne pas laisser les élèves dans l'incompréhension, surtout en zone d'éducation prioritaire, peut laisser les classes sur le bord de la littérature si les conduites lexicales se limitent à la catégorie traditionnelle de la définition qui donne la priorité à l'entrée référentielle.

Compte tenu de tout ce qui vient d'être dit, nous proposons dans la section suivante des pistes qui pourront être utilisées par les enseignants. Il ne s'agit donc pas d'un dispositif didactique finalisé, mais de directions didactiques indicatives.

\subsubsection{Quelques indications didactiques}

Dans ces directions, le style interactif de l'enseignant ou pattern interactif suggestif (Alincaï, Weil-Barais, Caillot, 2005) se révèle déterminant puisque c'est à travers lui que sont favorisées la participation et les verbalisations réflexives des élèves autour des mots du texte.

Les premiers types d'activités possibles que nous proposons gravitent autour de l'objectif suivant: s'emparer des faits lexicaux pour échanger et instaurer un rapport personnalisé aux mots du texte.

1) Une première phase pourrait commencer par la lecture intégrale du texte par l'enseignant qui donne accès à l'ensemble signifiant de celui-ci et offre la possibilité à chacun des élèves de s'en saisir et de l'interpréter. Il s'agirait ensuite de demander aux élèves de faire une liste de mots variés (noms, adjectifs, verbes, adverbes, expressions, etc.) ou de «bouts de texte» qui leur plaisent : des mots ou groupes de mots qu'ils connaissent bien, des mots ou groupes de mots incongrus ( «bizarres » ou « étonnants ») ou encore des mots ou groupes de mots qu'ils n'aiment pas. Cette façon de procéder les incite ainsi à ne pas se focaliser sur les mots simples et la catégorie des noms. Elle permet aussi d'établir un rapport d'intérêt individuel avec les mots ou groupes de mots qu'ils retiennent et qu'ils exposeront ensuite collectivement à la classe. 
2) Une seconde phase permettrait de constituer des groupes d'élèves à partir de la collecte réalisée dans la phase 1. Dans cette phase, l'enseignant pourrait guider ces élèves pour les amener à ce que le classement de la phase 1 soit d'abord justifié puis affiné et relié plus spécifiquement à l'œuvre lue. Ainsi, les élèves seraient amenés à associer par argumentation/tâtonnement l'ensemble des mots/groupes de mots/ «bouts de texte» recueillis soit aux personnages de l'œuvre, soit à ses dimensions imaginaire, anthropologique et/ou symbolique. Pour associer l'ensemble de la collecte à ces dimensions, l'enseignant devra conduire les élèves à relier leurs relevés à des mots-pivots (Chabanne, 2005, op.cit, Dupuy, 2009, op.cit) qui se rapportent à ces dimensions. Par exemple dans Archimémé, œuvre interrogée dans notre corpus, l'enseignant pourrait inciter ses élèves à rassembler l'ensemble des mots/groupes de mots/ « bouts de texte " trouvés qui se rapportent au sème /vieillesse/ dont font partie les UL voix fade et usée et rire grelottant. Pour La Petite fille du livre, toujours dans notre corpus, l'enseignant pourrait relier la collecte au sème/protection/ qui parcourt l'œuvre et l'image puisque la femme écrivain incarne dans les illustrations la figure maternelle protectrice jusqu'à pouvoir être assimilée à la représentation de La Vierge à l'enfant. À l'issue de cette seconde phase, les élèves pourront enfin garder leurs mots/groupes de mots et «bouts de texte» collectés dans leur carnet de lecture, ce qui pourrait donner lieu dans un prolongement à un écrit axiologique d'interprétation de l'œuvre.

3) Une autre direction didactique peut être proposée en fonction des œuvres lues. Elle consisterait à faire «vivre les mots par le corps »(Chabanne, Cellier, Dreyfus, 2006). Dans l'activité suivante, l'enseignant est amené à choisir parmi les mots sélectionnés par ses élèves un mot ou plusieurs mots (verbes fréquents, expressions) qui leur permettent d'exprimer leur sensibilité lexicale à travers le langage corporel. Ce type de proposition n'est pas à systématiser mais dépend bien de l'œuvre étudiée. Des traces sous forme de photos ou de vidéos pourront être ensuite exploitées pour un réinvestissement des mots dansés, mimés et dramatisés à destination d'un portfolio numérique. Ce portfolio pourrait être mis en relation avec le carnet de lecteur pour faciliter la mémorisation, favorisée par le rapport sensible aux mots, et donner la possibilité aux élèves de s'exprimer à nouveau, offrant ainsi un nouveau contexte d'utilisation pour un passage au vocabulaire actif.

Les seconds types d'activités concernent l'aspect syntagmatique des textes. Elles poursuivent l'objectif d'inciter les élèves à recourir au cotexte pour comprendre et interpréter un texte littéraire. Elle permet à la fois de faire observer aux élèves le lien qui existe entre lexique et syntaxe mais aussi d'entrer dans la compréhension/interprétation de l'œuvre comme lieu de rencontre entre deux vocabulaires, celui de l'auteur (donc du texte) et celui du lecteur. Très souvent, ce n'est pas la signification des mots qui posent problème aux élèves mais l'assemblage de ces mots dans le tissu textuel (métaphore, effets stylistiques recherchés). Dans notre corpus, on aurait pu imaginer une conduite lexicale de l'enseignant autour de l'UL voix fade et usée invitant les élèves à s'interroger sur le contexte d'usage des adjectifs (un vêtement usé et non une voix usée ; un gout fade et non une voix fade) pour repérer cette association métaphorique. Le tout étant là encore à relier à l'isotopie textuelle (Rastier, 1987/2009, op.cit.) de la vieillesse qui soutient l'interprétation de l'œuvre. C'est pour cette raison que nous insistons sur ce point car cette conduite est au cœur de notre entrée spécifique. L'aspect stylistique du vocabulaire en littérature est généralement très peu exploité par les enseignants non spécialistes, comme le montre le moment lexical analysé en début d'article à propos de cette UL.

\section{Conclusion}

À travers cet article nous avons pu rendre compte d'une partie de l'épaisseur et de la complexité de l'activité métalexicale en contexte littéraire, qu'elle porte sur les mots d'un texte ou sur des mots issus du commentaire que nous avons appelés mots de la réception. Cette épaisseur est liée d'une part aux différents traitements des mots (explication, définition et commentaires), d'autre part à l'imbrication des paroles des élèves et des enseignants qui constitue des mouvements discursifs conjoints (Jeanneret, 1988, op.cit.) dans lesquels sont mêlées l'interprétation singulière et l'interprétation collective qui font du sens un élément dynamique tel que l'envisage Rastier (2000).

À un autre niveau, les moments lexicaux objets de nos analyses constituent des espaces d'observation de gestes professionnels ajustés. Ces gestes professionnels que nous avons cherché à caractériser sont variés et ne peuvent répondre à une définition a priori, puisqu'ils correspondent à des pratiques de communautés discursives diverses (Jaubert, Rebière, Bernié, 2003, op.cit.) inscrites dans des contextes sociodiscursifs variés. D'où l'importance de décrire et analyser ce que les enseignants font avec leurs classes : quelles UL choisissent-ils de thématiser, avec quelles visées ? C'est donc bien l'analyse des données authentiques qui constitue un vivier réflexif pour les activités métalexicales en situation de lecture littéraire. 
Dans la spécificité qui nous occupe, la didactique a donc particulièrement intérêt à se nourrir de pratiques écologiques qui se montrent riches et que le chercheur par définition n'a pas à modifier. Nous en avons donné un aperçu dans cette analyse en procédant en quatre temps.

Nous nous sommes d'abord appuyées sur la description à grain fin des pratiques, ce qui nous a permis d'identifier certains gestes lexique convoqués par les enseignants, pour ensuite opérer une double translation: une première, sous la forme d'une typologie d'actions transférables à toute séance de lecture littéraire, une seconde sous la forme de stratégies lexicales qui pourraient être formulées auprès des élèves.

Nous avons enfin indiqué quelques pistes didactiques à partir du corpus restreint que nous avons donné à voir dans l'article mais qui donne déjà un aperçu des gestes lexique qu'il est possible de mobiliser à la croisée entre linguistique et compréhension/interprétation dans des séances de lecture littéraire.

\section{Références bibliographiques}

Anctil, D., Singcaster, M., Tardif M. (2018). Pratiques d'enseignement du lexique au primaire au Québec. La Lettre de l'AIRDF, 64, 19-25.

Barthes, R., (1973). Le plaisir du texte. Paris : Seuil.

Borillo, A. (1985). Discours ou métadiscours? DRLAV, 32, 47-61.

Borillo, A. (2004). Les adverbes d'opinion forte » selon moi, à mes yeux, à mon avis,... : point de vue subjectif et effet d'atténuation. Langue française, 142,31-40.

Bruner, J. S. (1983). Le Développement de l'enfant : savoir-faire, savoir dire. Paris : P.U.F.

Bucheton, D., Dezutter, O. (dir.). (2008a). Le développement des gestes professionnels dans l'enseignement du français : un défi pour la recherche et la formation. Louvain : De Boeck.

BuCHETON, D. et Soulé, Y. (2009). Les gestes professionnels et le jeu des postures de l'enseignant dans la classe : un multi-agenda de préoccupations enchâssées. Éducation et didactique, vol 3 - n³, 29-48.

Cavalla, C. (2018). Lexique transdisciplinaire et enseignement aux étudiants allophones. Agnès Tutin; Marie-Paule Jacques. Lexique transversal et formules discursives des sciences humaines, Grenoble : ISTE Editions, 191-214.

Chabanne, J.-C. (2005). Lexique et lecture littéraire: mettre le travail sur le lexique au service de l'interprétation/compréhension des textes littéraires. Communication aux journées Enseignement du lexique, mars 20015, Lyon : INRP. Texte non publié.

Chabanne, J.C., Cellier, M., Dreyfus, M. (2006). « Les enjeux lexicaux de la lecture littéraire ». Dans F. Grossmann et S. Plane (dir.). Les apprentissages lexicaux: Lexique et production verbale. Actes des journées INRP/LIDILEM, Lyon : Presses universitaires du Septentrion, p.85-101.

Dupuy, C. (2009). Le français tel qu'on l'enseigne. Étude des gestes professionnels de maîtres faisant lire un texte de littérature jeunesse au cycle 3 » LIRDEF/UM. UPVM. Thèse https://tel.archives-ouvertes.fr/tel-00464095/document.

Dupuy, C., Genre, S., (2020). Pratique littéraire du lexique/vocabulaire : les sens construits dans les moments lexicaux. Un texte dans la classe. Pratique d'enseignement de la littérature au cycle 3 en France. B. Louichon (dir.). Berne : Peter Lang. Collection Théocrit.

Flanagan, J.C. (1954). The critical incident technique » [La technique de l'incident critique]. Psychological Bulletin, vol.51 (4), 327-358.

François, F. (1990). La communication inégale: heurs et malheurs de l'interaction verbale. Paris : Delachaux et Niestlé.

Garcia-Debanc, C. (2013). Les aides lexicales à la rédaction de textes : panorama historique et essai de typologie », in Garcia-Debanc, C., Masseron, C., Ronveaux, C. (éds), Enseigner le lexique. Presses universitaires de Namur, 273-300.

Gaulmyn, M.M. (1987). Reformulation et planification discursive. J. Cosnier et C. Kerbrat-Orrecchioni, Décrire la conversation, PU de Lyon, 167-198.

Genre, S. (2015). La glose à thème lexical en situation littéraire au cycle trois de l'école primaire française : entre épisodes métadiscursifs d'élucidation du sens et figures de l'ajout, un nœud de l'intervention didactique, un enjeu pour la formation d'enseignant. LIRDEF/UM.UPVM. https://tel.archives-ouvertes.fr/tel-01320127

Genre, S. (2018). Vocabulaire et littérature : enjeux didactiques du paradigme désignationnel dans une séance de lecture littéraire. SHS Web of Conferences 46, $07011 \mathrm{https} / / /$ doi.org/10.1051/shsconf/20184607011. Congrès Mondial de Linguistique Française - CMLF 2018.

Graves, M.-F. (2006). The Vocabulary Book. Learning and Instruction. New York, Teachers College : Columbia University.

Grossmann, F. (2011). Didactique du lexique : état des lieux et nouvelles orientations. Pratiques, 149-150, 163-183.

Gülich, E., Kotschi,T.(1987). Les actes de reformulation dans la consultation «La dame de Caluire. Bange (Ed.), L'analyse des interactions verbales. La dame de Caluire ». Berne : Peter Lang, 15-81.

Halté, J.-F., (1989). Discours explicatif : état et perspective de la recherche. Repères, 77,95-109.

Hudelot, C. (2001).Explication, explicitation, justification et mouvements discursifs chez le jeune enfant. Colloque International L'explication : enjeux cognitifs et communicationnels. Paris : Université de la Sorbonne.

Jacques, F. (1993). Interprétation et textualités. Comprendre et interpréter. J. Greisch (dir). Paris : Beauchesne. 
Jaubert, M., Rebière, M., Bernié, J.-P. (2003). L'hypothèse « communauté discursive » : d'où vient-elle ? Où va-telle ? Les cahiers THÉODILE, 4, 51-80.

Jeanneret, T. (1988).Analyse hiérarchique et fonctionnelle du discours : co-énonciation et mouvement discursif. TRANEL, 13, 15-42.

Kerbrat-Orecchioni, C. (1990/1998). Les interactions verbales. I/ Approche interactionnelle et structure des conversations. Paris : Armand Colin.

Kerbrat-Orecchioni, C. (2001/2008). Les actes de langage dans le discours. Théorie et fonctionnement. Paris : Armand Colin.

Langlade, G. (2008). Activité fictionnalisante du lecteur et dispositif de l'imaginaire. M.Roy, M.Brault, S. Brehm. (dir). Formation des lecteurs. Formation de l'imaginaire, Montréal : Université du Québec, 45-65.

Lüdi, G. (1991). Métaphore et travail lexical. TRANEL, 17, 17-49.

Mortureux, M.-F. (1997/2013). La lexicologie entre langue et discours. Paris : Armand Colin.

Nonnon E. (2012). La didactique du français et l'enseignement du vocabulaire dans vingt ans de revues de didactique $\mathrm{du}$ français langue première. Repères, 46, 33-72.

Plane, S. (2005). Les activités définitionnelles au service des apprentissages lexicaux. Pratiques, 125-126,115-123.

Polguère, A. (2016). Lexicologie et sémantique lexicale. Notions fondamentales. Les Presses Universitaires de Montréal, $3^{\circ}$ édition.

Rastier, F. (1987/2009). Sémantique interprétative. Paris : PUF.

Rastier, F. (2000). Problématiques du sens et de la signification. J.-M. Barbier, O. Galanatu (dir.). Signification, sens, formation. Paris : PUF, 5-24.

Rouxel, A. et Langlade, G. (2004). Qu'entend-on par lecture littéraire ? La lecture et la culture littéraire au cycle des approfondissements. Versailles : CRDP.

Tauveron, C., (2005). Droits du texte et droits des jeunes lecteurs : un équilibre instable. Rouxel et Langlade. (dir.) $L e$ sujet lecteur : lecture subjective et enseignement de la littérature. Rennes. PUR.

Theureau, J. (2006). Le cours d'action. Méthode développée. Toulouse. Octares.

Tremblay, O. (2004) : Pour un apprentissage structuré de l'enseignement-apprentissage du lexique. Calaque, E. et David, J., Didactique du lexique. Contexte, démarches, supports. Louvain :De Boeck Université.

Tremblay, O, Anctil, et D., Perron, V. (2016). Synthèse sur la compétence lexicale. Colloque AIRDF, Montréal. Non publié.

Tremblay, O. (2017). Entrer dans le lexique par la porte des sentiments. Correspondance, 22(6). https://correspo.ccdmd.qc.ca/index.php/document/entrer-dans-le-lexique-par-la-porte-des-sentiments/

Vergnaud, G. (2001). Forme opératoire et prédicative de la connaissance. Portugais J. (éd.), La notion de compétence en mathématiques. Actes du colloque GDM-2001.Montréal: UQAM. http://smf.emath.fr/Enseignement/TribuneLibre/EnseignementPrimaire/ConfMontrealmai2001.pdf.

Volteau, S. Garcia-Debanc, C. (2008).Gérer les reformulations: un geste professionnel. Influence des objets enseignés sur les types de reformulation. D. Bucheton, O. Dezutter. (dir.). Le développement des gestes professionnels dans l'enseignement du français. Un défi pour la recherche et la formation. Louvain: de Boeck, 191-212.

Volteau, V., Garcia-Debanc, C., Panissal, P. (2010). Les reformulations définitoires dans les interactions scolaires, in Publifarum Autour de la définition http://www.publifarum.farum.it/appel.php. Université de Gênes.

Ailincaï, R.,Weil-Barais, Caillot, M. (2005). De l'étude des interactions parents-enfants dans un contexte muséal scientifique à une proposition d'intervention innovante auprès des parents. Les Quatrièmes rencontres de l'ARDIST, 5-12.

Wilks, C., Meara, P., (2002). Untangling Word Webs: Graph Theory and the Notion of Density in Second Language Word Association Networks. Second Language Research, 18(4), 303-324. 